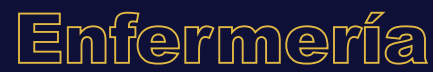

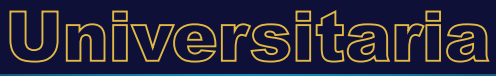

\section{Caracterización de las investigaciones de tesis de posgrado en enfermería}

\section{Nursing postgraduate researching thesis characterizations}

\section{Caracterização da pesquisa de teses de pós-graduação em enfermagem}

\section{Hernández-Márquez ${ }^{a}$, T. Ojeda-Chacón ${ }^{b}$, P.I. Torres-Paz ${ }^{\text {c }}$ E. Arizmendi-Jaime ${ }^{\mathrm{d}^{*}}$}

ORCID:

${ }^{\mathrm{a}} \underline{0000-0003-3801-4435} \quad \quad \mathrm{~d} \underline{0000-0002-75657715}$

${ }^{\mathrm{b}} 0000-0001-8431-1014 \quad{ }^{\mathrm{e}} \underline{0000-0002-6313-1687}$

Universidad Autónoma del Estado de Morelos, Facultad de Enfermería, Cuernavaca, Morelos, México

Recibido: 10 marzo 2019

Aceptado: 12 julio 2020

\section{RESUMEN}

Introducción: En la elaboración de tesis de posgrado los estudiantes se acercan a la investigación y adquieren competencias en sus campos disciplinares. El análisis de sus características permite reconocer tendencias respecto a las decisiones para el desarrollo del conocimiento científico e identificar áreas de oportunidad en la generación del conocimiento. objetivo: Identificar las características de los trabajos de tesis realizados por egresados de maestría y especialidad en enfermería.

Métodos: Estudio retrospectivo, transversal, descriptivo. Se analizaron 144 tesis presentadas entre 2013 a 2018, se determinó la correspondencia del tema con la LGAC y programa cursado, el objeto de estudio, metodología utilizada y tipo de estudio realizado. 
Resultados: Los temas de la especialidad en administración corresponden 91.7\% con administración, los de especialidad en atención al adulto en estado crítico tienen una correspondencia del 86\% con la atención integral institucional, en el caso de maestría $51.1 \%$ corresponde con temas de cuidado y promoción de la salud. El objeto de estudio predominante fue el actuar enfermero (52.8\%), conocimientos de los profesionales (60\%) y calidad de atención (32\%). 91\% utilizó metodología cuantitativa, prevalecieron en $88.9 \%$ los estudios observacionales.

Discusión: Los resultados muestran la necesidad de ampliar la formación en investigación de estudiantes y docentes del posgrado en Enfermería.

Conclusión: Los temas abordados en las tesis tienen correspondencia con los objetivos de los programas. La metodología más utilizada fue la cuantitativa y el estudio observacional, con estudios de enfermería centrados en intervenciones para el mejoramiento de los problemas que enfrenta el paciente y los profesionales.

Palabras clave: Tesis académica como asunto; educación de posgrado en enfermería; investigación en enfermería; México.

\section{ABSTRACT}

Introduction: While producing their postgraduate thesis, students get closer to researching activities and acquire diverse competencies in their discipline fields. The analysis of these trends allows a better identification of the decisions related to areas of opportunity and the development of scientific knowledge.

Objective: To identify the characteristics of the thesis works produced by graduate students from the Masters in Nursing and the Specialty Nursing programs.

Methods: This is a retrospective, transversal, and descriptive study. 144 thesis works submitted during the period 2013-2018 were analyzed. The degree of correspondence between the topics presented in the submitted works and the current Lines of Generation and Application of Knowledge (LGAK) was assessed.

Results: The topics related to management had a correspondence of $91.7 \%$ with the management LGAK; the topics related to older adult in critical status attention had a correspondence of $86 \%$ with the institutional integral attention LGAK, and regarding the Masters in Nursing program specifically, a 51.1\% correspondence was found with the topics of healthcare and health promotion. The main objects of study were: the performance of the nursing personnel (52.8\%); the knowledge of nursing professionals (60\%); and the quality of the attention (32\%). 91\% of the thesis works followed a quantitative approach, with $88.9 \%$ being observational.

Discussion: The results suggest the need to consider enhancing the scope of researching formation among both the students and academic staff in this postgraduate program.

Conclusion: The topics addressed in the submitted works showed sufficient correspondence with the objectives of the programs. The most frequently used methodological approach was the quantitative-observational. Nursing studies focused on interventions to improve the situations which both patients and health professionals face were the most prevalent. Keywords: Academic dissertation as topic; education, nursing, graduate; nursing research; Mexico. 


\section{RESUMO}

Introdução: $\mathrm{Na}$ elaboração de teses de pós-graduação, os estudantes aproximam-se da pesquisa e adquirem competências em seus campos disciplinares. A análise de suas caraterísticas permite reconhecer tendências a respeito das decisões para o desenvolvimento do conhecimento científico e identificar áreas de oportunidade na geração do conhecimento. Objetivo: Identificar as características dos trabalhos de teses realizados por estudantes de graduação de mestrado e especialidade em enfermagem.

Métodos: Estudo retrospectivo, transversal, descritivo. Foram analisadas 144 teses apresentadas entre 2013 e 2018, determinou-se a correspondência do tema com a LGAC e programa cursado, o objeto de estudo, a metodologia utilizada e o tipo de estudo realizado. Resultados: Os temas da especialidade em administração correspondem 91.7\% a administração, os de especialidade em atenção a adultos em estado crítico têm uma correspondência de $86 \%$ à atenção integral institucional e no caso de mestrado, 51.1\% correspondem a temas de cuidado e promoção da saúde. O objeto de estudo predominante foi a atuação do enfermeiro (52.8\%), conhecimentos dos profissionais (6o\%) e qualidade de assistência (32\%). 91\% utilizou metodologia quantitativa, os estudos observacionais prevaleceram em $88.9 \%$.

Discussão: Os resultados mostram a necessidade de ampliar a formação em pesquisa de estudantes e docentes de pós-graduação em Enfermagem.

Conclusão: Os temas abordados nas teses correspondem aos objetivos dos programas. A metodologia mais utilizada foi a quantitativa e o estudo observacional, com estudos de enfermagem centrados em intervenções para a melhoria dos problemas que enfrenta o paciente e os profissionais.

Palavras chave: Dissertações acadêmicas como assunto; educação de pós-graduação em enfermagem; pesquisa em enfermagem; México.

\section{INTRODUCCIÓN}

Eltrabajo detesis forma parte delas más antiguas tradiciones universitarias de Occidente. Borsingerde Montemayor ${ }^{1}$ la define como: un texto cuidadosamente elaborado, que emplea a menudo un discurso retórico en alto grado; es una comunicación escrita que implica un largo proceso de elaboración y maduración, de lugar y tiempo diferido entre el escritor y su lector.

En la actualidad, la escritura y defensa de la tesis es un requisito para la conclusión de estudios de posgrado que permite iniciar al estudiante en el campo de la investigación científica², conlleva un proceso de cambio que afecta los conocimientos, modifica las capacidades y habilidades adquiridas previamente, así como las actitudes y sentimientos del autor mediante un proceso de aprendizaje implícito ${ }^{3}$, representa una oportunidad para el desarrollo de pensamiento reflexivo y crítico ${ }^{4}$, impulsa el desarrollo de habilidades cognitivas, actitudinales y éticas ${ }^{5}$, así como también la expresión de aquello que fue particularmente significativo en la trayectoria del estudiante.

Según su temática, se inserta en una Línea de Generación y Aplicación del Conocimiento (LGAC), definida por el Consejo Nacional de Ciencia y Tecnología (CONACYT) como campos temáticos en los que confluyen las trayectorias de las investigaciones de los profesores(as) integrantes del Núcleo Académico Básico de un Programa de Posgrado y el trabajo de los y las estudiantes, desde una 
perspectiva sistémica de generación de nuevo conocimiento o su aplicación ${ }^{6}$. Estas líneas definen la naturaleza del Programa de Posgrado con base en el objeto de estudio y el perfil de egreso del estudiante; asimismo, constituyen la base fundamental para la generación y aplicación de nuevo conocimiento científico, útil para la solución de problemáticas en la disciplina desde el posgrado que promueve en los estudiantes el desarrollo de una actitud colaborativa y de compromiso, así como un cambio hacia relaciones y formas de trabajo. El análisis de la productividad científica, en este caso representada por las tesis de posgrado, favorece un acercamiento a la comprensión de las decisiones individuales y colectivas para el desarrollo del conocimiento científico, lo que permite reconocer sus tendencias, su interés, los objetos de análisis y los enfoques metodológicos más utilizados?.

En México, los programas de posgrado contemplan la realización y defensa de una tesis como producto de un trabajo de investigación propio y original ${ }^{8}$. En enfermería se enfoca hacia la comprensión de los fenómenos que se presentan en la práctica profesional diaria ${ }^{9}$, relacionados con el cuidado desde la perspectiva técnica, administrativa o cognitiva ${ }^{4}$ se reconocen cuatro modelos de investigación: intervencionista, correlacional, observacional descriptivo y sociológico, con la utilización de métodos mayoritariamente cuantitativos, sobre los cualitativos y mixtos ${ }^{10}$.

Las características de las tesis de posgrado en enfermería se han estudiado en diversos países y momentos. A partir de su análisis se han detectado algunos aspectos que pueden ser mejorados; Bispo-Hirano et al..$^{11}$ encontraron en Brasil que la mayoría de las tesis corresponden a estudios descriptivos y utilizan la taxonomía de la North American Nursing Diagnosis Association (NANDA) con escasa utilización práctica de sus resultados. Si bien es cierto que el mayor interés en la investigación se dirige a la identificación de problemas prioritarios de salud, sus determinantes y condicionantes con el fin de mejorar el cuidado de enfermería ${ }^{12}$, en las tesis se observa pobre análisis que evidencie el desarrollo de pensamiento reflexivo y crítico, mismo que Nájera y Castrillón ${ }^{13}$ consideran necesario para la elaboración de juicios eficientes durante las situaciones clínicas. Esta característica es reforzada por Echeverría ${ }^{14}$, en cuya investigación destaca que el pensamiento crítico no es utilizado en el manejo de las enfermeras en función asistencial. A su vez, Franco et al. ${ }^{4}$ señalan que el nivel de impacto de las tesis de posgrado es bajo, por su evidencia insuficiente de resultados.

Con respecto a las temáticas, las que se abordan en las tesis de posgrado en enfermería suelen ser similares en diversos contextos. Souza ${ }^{15}$ encontró que las tesis de maestría en enfermería en Brasil tienen como tendencia su vinculación con estilos de vida saludables, seguido de gestión, gerencia y administración. Por su parte, Silvan-Scochi et al. ${ }^{16}$ encontraron que la mayoría de las tesis de doctorado realizadas en Brasil se relacionan con la atención en salud y enfermería, presentan deficiencias en el conocimiento sobre fundamentos teóricos y filosóficos del cuidado, así como de historia y ética de la enfermería; predominan los estudios descriptivos y/o exploratorios. En el mismo país, PartezaniRodrigues et al. ${ }^{17}$ al analizar las tesis doctorales de enfermería encontraron que el máximo interés de investigación se centra en la evaluación de conocimientos del personal de enfermería sobre aspectos relacionados con la práctica profesional. Una característica común es que, a pesar de la importancia de la elaboración de tesis en posgrado, los estudiantes enfrentan serios problemas que limitan sus intereses, objetivos y logros, ${ }^{18}$ lo que afecta al desarrollo y crecimiento de la disciplina ${ }^{19}$.

Por lo anterior, se realizó una revisión de las tesis de posgrado de la Facultad de Enfermería (FE) de la Universidad Autónoma del Estado de Morelos (UAEM) para establecer la cantidad producida e identificar tendencias, intereses, metodología y temas involucrados en su elaboración, con el fin de reconocer áreas de oportunidad para la mejora del campo de investigación en enfermería, así como impulsar, fortalecer y acompañar el trabajo de los estudiantes en el avance de su proyecto, 
ya que en estudios de posgrado la escritura de la tesis representa una oportunidad de aprendizaje enmarcada en una forma de escribir propio de una disciplina y el inicio de la participación activa de los posgraduados en ella ${ }^{20}$.

En la Facultad de Enfermería de la UAEM, el posgrado en enfermería inicia en $2011^{21}$ con programas que proporcionan a los egresados competencias profesionales específicas en un área determinada del conocimiento enfermero y desarrollan habilidades para la docencia y la investigación. Con el propósito de dar sentido y significado académico a la investigación, en la FE se desarrollan dos LGAC que se centran en temas propios de la disciplina en dos áreas principales: atención integral institucional que comprende la investigación realizada en torno a la gestión y calidad del cuidado, así como los aspectos relacionados con éste dentro de las instituciones hospitalarias, lo que incluye investigación sobre calidad de atención, competencias de los profesionales en enfermería y gestión del cuidado. La otra LGAC denominada cuidado y promoción de la salud engloba los productos de investigación sobre temas relativos a la atención de enfermería en salud comunitaria, con principal énfasis en el desarrollo de intervenciones a nivel comunitario que tienen como fin el mejoramiento de las condiciones de salud de las comunidades, especialmente en la prevención y control de padecimientos crónico-degenerativos. Incluye también investigaciones en adolescentes y en estudiantes de enfermería.

Cabe destacar que ambas LGAC fomentan el desarrollo de habilidades y destrezas que permiten al egresado participar en investigaciones científicas para incrementar el conocimiento en el ámbito de la salud y desarrollar la capacidad de investigación en el alumno ${ }^{22}$. En estos trabajos se utilizan metodología cualitativa y cuantitativa ${ }^{23}$ (esta última es la más seleccionada), se intenta dar respuesta a problemáticas encontradas ${ }^{24}$, mayoritariamente se recurre al enfoque positivista y en menor proporción a la filosofía humanística.

El objetivo del presente artículo es identificar las características de las tesis realizadas por egresados de los programas de maestría y especialidad en enfermería de la Facultad de Enfermería de la Universidad Autónoma del Estado de Morelos, México, con el fin de dar respuesta a la pregunta: ¿cuáles son las características (teórico-conceptuales, metodológicas, nivel del conocimiento, áreas temáticas) y tendencias respecto a estas características seleccionadas de las tesis elaboradas por estudiantes de posgrado en enfermería?

Ésta permitirá obtener y sistematizar datos sobre el objeto de interés para los estudiantes al realizar su investigación; las diferencias que se presentan en las tesis elaboradas por alumnos de especialidad y maestría; la correspondencia de los temas elegidos con los objetivos de los programas e identificar áreas de oportunidad en la realización de tesis con la finalidad de fortalecer los programas de posgrado por medio de la generación de evidencia científica, orientar las tesis al abordaje de temas más relevantes para la disciplina y una mejor asesoría a los estudiantes para la adecuada toma de decisiones que afectan el área y los sujetos de interés.

\section{METODOLOGÍA}

Se realizó un estudio retrospectivo, transversal, descriptivo, se analizaron de forma individual todos los trabajos de tesis presentados para la obtención del grado académico por egresados de maestría y especialidad en enfermería en sus dos opciones terminales: 1) atención al adulto en estado crítico; 2) administración y gestión del cuidado de la salud, de 2013 a 2018.

Para la obtención de información se recurrió a los registros de los títulos de tesis de la jefatura de posgrado de la FE, se incluyeron todas las tesis presentadas en ese periodo, por lo que no se 
consideraron criterios de inclusión, exclusión o eliminación. En cada tesis se evaluaron las variables: grado de la tesis (maestría y especialidad), LGAC correspondiente (Atención integral institucional y, cuidado y promoción de la salud), características teórico-conceptuales, metodológicas: Área de estudio (Administración, Cuidado crítico, Enfermería comunitaria), Metodología (Cuantitativa o cualitativa), Diseño de la investigación (Estudios experimentales u observacionales), Objeto de estudio (Actuar enfermero, Administración de servicios, Autocuidado, Conducta de riesgo, Educación a pacientes, Participación comunitaria, Procesos patológicos, Riesgos para la salud, Sentimiento enfermero y sentimiento de pacientes), sujetos de interés (Personal de enfermería, pacientes hospitalizados, pacientes crónicos, estudiantes, adolescentes, neonatos, familiares, madres, adultos mayores, embarazadas, escolares) y Dominio de conocimiento (diagnóstico, intervención, resultados). Cada una de estas variables fue registrada en una cédula diseñada para este fin. Se manejaron variables dicotómicas, colocando el valor de 1 cuando cumplía con la característica y o cuando no la presentaba. La cédula fue validada por consenso de expertos docentes de la FE. Los datos colectados fueron tabulados en el programa MS Excel 2016 y analizados con el paquete estadístico stata ${ }^{14}$.

La información utilizada en la investigación es de dominio público, la recopilación de datos no involucró directamente a seres humanos, no requirió aprobación por parte del Comité de Ética en Investigación de la Universidad, ni de participantes, como lo indica el Consejo Nacional de Salud. Se omitió el nombre del autor y director de la tesis para respetar su anonimato.

\section{RESULTADOS}

Se incluyó un total de 144 tesis presentadas por egresados de programas de posgrado de 2013 a 2018. Del total, 61.1\% correspondieron al nivel maestría, 30.5\% a la Especialidad en atención al adulto en estado crítico y $8.3 \%$ a la Especialidad en administración y gestión del cuidado. Con relación a las LGAC, se observa que $48.9 \%$ de las tesis de maestría y $74.2 \%$ de especialidad se insertan en la LGAC de Atención integral institucional, el resto en la línea de Cuidado y promoción de la salud (Tabla 1).

\begin{tabular}{|c|c|c|c|c|c|c|}
\hline \multirow[t]{3}{*}{ Programa } & \multicolumn{4}{|c|}{ LGAC } & \multicolumn{2}{|c|}{ Total } \\
\hline & \multicolumn{2}{|c|}{$\begin{array}{l}\text { Atención integral } \\
\text { institucional }\end{array}$} & \multicolumn{2}{|c|}{$\begin{array}{l}\text { Cuidado y promoción } \\
\text { de la salud }\end{array}$} & \multirow[b]{2}{*}{$\mathbf{n}$} & \multirow[b]{2}{*}{$\%$} \\
\hline & $\mathbf{n}$ & $\%$ & $\mathbf{n}$ & $\%$ & & \\
\hline $\begin{array}{l}\text { Especialidad en administración y } \\
\text { gestión del cuidado }\end{array}$ & 11 & 91.7 & 1 & 8.3 & 12 & 8.3 \\
\hline $\begin{array}{l}\text { Especialidad en atención al adulto en } \\
\text { estado crítico }\end{array}$ & 38 & 86.3 & 6 & 13.6 & 44 & 30.5 \\
\hline Maestría en enfermería & 43 & 48.9 & 45 & 51.1 & 88 & 61.1 \\
\hline Total & 92 & 63.9 & 52 & 36.1 & 144 & 100 \\
\hline
\end{tabular}

Las tesis de los egresados de la especialidad en administración y gestión del cuidado de la salud corresponden 91.7\% a administración; las tesis de la especialidad de Atención al adulto en estado crítico corresponden $79.5 \%$ al ámbito de cuidado a pacientes en estado crítico. En lo referente a las tesis de maestría, que tiene dos áreas de énfasis, $48.9 \%$ conciernen al área administrativa y $51.1 \%$ a la de enfermería comunitaria (Tabla 2). 
Tabla 2. Correspondencia del área de tesis con el Programa (Porcentajes, $N=144$ )

\begin{tabular}{|c|c|c|c|c|c|}
\hline \multirow[b]{3}{*}{$\begin{array}{l}\text { Especialidad en administración } \\
\text { y gestión del cuidado }\end{array}$} & \multicolumn{3}{|c|}{ Área de Tesis } & \multicolumn{2}{|c|}{ Total } \\
\hline & Administración & $\begin{array}{l}\text { Cuidado } \\
\text { crítico }\end{array}$ & $\begin{array}{l}\text { Enfermería } \\
\text { Comunitaria }\end{array}$ & & \\
\hline & 91.7 & & 8.3 & 12 & 100 \\
\hline $\begin{array}{l}\text { Especialidad en atención al } \\
\text { adulto en estado crítico }\end{array}$ & 6.8 & 79.5 & 13.6 & 44 & 100 \\
\hline Maestría en enfermería & 48.9 & & 51.1 & 88 & 100 \\
\hline
\end{tabular}

En relación con la metodología utilizada, 91\% correspondieron a estudios cuantitativos; en lo referente al diseño de investigación, se tiene una clara tendencia a realizar estudios observacionales (88.9\%).

El objeto de estudio fue predominantemente el actuar enfermero en todos los programas (52.8\%), particularmente en el programa de especialidad en atención al adulto en estado crítico, conocimientos de los profesionales (60\%) y calidad de atención (32\%). En orden decreciente, la investigación sobre sentimientos de pacientes representó $11.1 \%$ y el estudio de conductas de riesgo 10\%. Existe diferencia en los temas de elección de acuerdo a los programas. El programa de maestría cubre un mayor número de áreas y las tesis de éste exploran en mayor medida aspectos vinculados con la prevención y promoción de la salud en tanto que abordan autocuidado, conductas de riesgo, educación a pacientes y participación comunitaria, mientras la especialidad en administración y gestión del cuidado de la salud se concentra solo en cinco, todas relacionadas con temas administrativos (Tabla 3).

\section{Tabla 3. Objeto de estudio en las tesis de posgrado según programa de posgrado}

\begin{tabular}{|c|c|c|c|c|c|c|c|c|}
\hline \multirow[t]{3}{*}{ Área de interés } & \multicolumn{4}{|c|}{ Especialidad de enfermería } & \multicolumn{2}{|c|}{$\begin{array}{l}\text { Maestría en } \\
\text { Enfermería }\end{array}$} & \multicolumn{2}{|c|}{ Total } \\
\hline & \multicolumn{2}{|c|}{$\begin{array}{c}\text { Administración } \\
\text { y Gestión del } \\
\text { Cuidado }\end{array}$} & \multicolumn{2}{|c|}{$\begin{array}{l}\text { Atención al } \\
\text { adulto en } \\
\text { estado crítico }\end{array}$} & \multirow[b]{2}{*}{$\mathbf{n}$} & \multirow[b]{2}{*}{$\%$} & \multirow[b]{2}{*}{$\mathbf{n}$} & \multirow[b]{2}{*}{$\%$} \\
\hline & $\mathbf{n}$ & $\%$ & $\mathbf{n}$ & $\%$ & & & & \\
\hline Actuar enfermero & 6 & 50.0 & 29 & 65.9 & 41 & 46.6 & 76 & 52.8 \\
\hline Administración de servicios & 3 & 25.0 & & & 1 & 1.1 & 4 & 2.8 \\
\hline Autocuidado & & & 1 & 2.8 & 4 & 4.6 & 5 & 3.5 \\
\hline Conducta de riesgo & & & 3 & 6.8 & 11 & 12.5 & 14 & 9.7 \\
\hline Educación a pacientes & 1 & 8.3 & & & 1 & 1.1 & 2 & 1.4 \\
\hline Participación comunitaria & 1 & 8.3 & & & 3 & 3.4 & 4 & 2.8 \\
\hline Procesos patológicos & & & 1 & 2.3 & & & 1 & 0.7 \\
\hline Riesgos para la salud & & & 4 & 9.1 & 6 & 6.8 & 10 & 6.9 \\
\hline Sentimiento enfermero & & & 3 & 6.8 & 9 & 10.2 & 12 & 8.3 \\
\hline Sentimiento de pacientes & 1 & 8.3 & 3 & 6.8 & 12 & 13.6 & 16 & 11.1 \\
\hline Total & 12 & 100 & 44 & 100 & 88 & 100 & 144 & 100 \\
\hline
\end{tabular}


Los sujetos de mayor interés al realizar la investigación de los egresados fue el personal de enfermería con 35\%, seguido de pacientes hospitalizados y crónicos con 18\%. En el área de investigación en la comunidad, los grupos con mayor interés fueron los estudiantes universitarios (9\%) y los adolescentes (8\%), especialmente en lo referente a aspectos de sexualidad.

De acuerdo a los tres dominios de conocimiento de enfermería, las tesis muestran estudios de intervención en 51.4\%, especialmente los correspondientes a las áreas de administración y atención al adulto en estado crítico, mientras que las tesis de maestría muestran diagnósticos en 57.9\%, especialmente a nivel comunitario. Pocos son los trabajos que abordan el dominio de los resultados correspondiendo sólo 1.4\% del total de las tesis (Tabla 4).

\begin{tabular}{|c|c|c|c|c|c|c|c|c|}
\hline \multirow[t]{2}{*}{ Programa } & \multicolumn{6}{|c|}{ Dominios de conocimiento de enfermería } & \multicolumn{2}{|c|}{ Total } \\
\hline & \multicolumn{2}{|c|}{ Diagnósticos } & \multicolumn{2}{|c|}{ Intervenciones } & \multicolumn{2}{|c|}{ Resultados } & \multirow[b]{2}{*}{$\begin{array}{c}\mathbf{n} \\
12\end{array}$} & \multirow[b]{2}{*}{$\begin{array}{c}\% \\
100\end{array}$} \\
\hline $\begin{array}{l}\text { Especialidad en administración } \\
\text { y gestión del cuidado }\end{array}$ & $\begin{array}{c}\mathbf{n} \\
3\end{array}$ & $\begin{array}{c}\% \\
25.0\end{array}$ & $\begin{array}{c}\mathbf{n} \\
9\end{array}$ & $\begin{array}{c}\% \\
75.0\end{array}$ & $\mathbf{n}$ & $\%$ & & \\
\hline $\begin{array}{l}\text { Especialidad en atención al } \\
\text { adulto en estado crítico }\end{array}$ & 14 & 31.8 & 29 & 65.9 & 1 & 2.3 & 44 & 100 \\
\hline Maestría en enfermería & 51 & 57.9 & 36 & 41.0 & 1 & 1.1 & 88 & 100 \\
\hline Total & 68 & 47.2 & 74 & 51.4 & 2.0 & 1.4 & 144 & 100 \\
\hline
\end{tabular}

En las tesis con intervenciones se realizaron mediciones basales, se manipuló la variable de interés y se valoró el cambio de la misma posterior a la intervención, de las cuales surgieron propuestas de mejora para las instituciones en donde se realizó la investigación.

\section{DISCUSIÓN}

Se torna imprescindible la necesidad de estudiar con profundidad la productividad científica que se genera desde los programas de posgrado en enfermería; determinar el nivel de fundamentación teórica y metodológica, además de evaluar el desarrollo del pensamiento crítico; así como definir el tipo de pensador que requiere la formación académica de posgrado en Enfermería 4 . Todo ello para lograr, además de la generación del conocimiento, el desarrollo de procesos afectivos en los alumnos de posgrado, que de acuerdo con Saltos et al.5, desarrollan habilidades cognitivas, habilidades actitudinales y éticas valorativas, mismas que son generadas mediante la investigación y que son necesarias en el campo profesional de la enfermería.

En este estudio se puede observar que el número de tesis concluidas por programa guarda relación con el tiempo de creación y con el número de alumnos egresados de cada uno. La relación entre programas y LGAC refleja el modelo hegemónico predominante en el cual se magnifica la atención clínica institucional, lo que corresponde a una demanda de personal especialista en áreas clínicas de enfermería a la que la Universidad da respuesta.

El programa de maestría en enfermería abre la opción al estudio del cuidado y promoción de la salud al tener un área con énfasis en enfermería comunitaria, lo que propicia la elaboración de tesis con perspectiva de salud comunitaria.

Los resultados obtenidos muestran que el sujeto de interés predominante es el actuar enfermo, resultados que coinciden con estudios elaborados previamente que muestran que en enfermería 
destacan estudios sobre personal de enfermería. De igual forma, los resultados de esta investigación son similares a los obtenidos por Silvan-Scochi et al. ${ }^{16}$ en cuanto a un predominio de estudios descriptivos, observacionales, lo que pudiera estar condicionado por limitaciones de tiempo, técnicas, económicas e institucionales para el desarrollo de estudios experimentales como trabajos de tesis.

En cuanto a la metodología utilizada, a diferencia de lo encontrado por Marocco-Duran y Pellegrino-Toledo ${ }^{24}$ en Brasil, se observa en este grupo de tesis incluidas una mayor tendencia a la investigación cuantitativa con poca incursión en la cualitativa, misma que se desarrolla especialmente sobre los aspectos que conectan la práctica de la enfermería con los contextos políticos y sociales ${ }^{23}$. Dado el carácter social de la enfermería, sería interesante considerar el uso de la metodología cualitativa en las investigaciones de tesis, lo cual fortalecería la comprensión de los significados de fenómenos relacionados al cuidado profesional posibilitando el desarrollo de los constructos teóricos en enfermería, además de aportar valor científico en beneficio de la disciplina y la sociedad en general y contribuir al progreso de la ciencia ${ }^{19}$.

Asimismo, se encuentra coincidencia con lo reportado por Partezani-Rodrigues et al. ${ }^{17}$ en su estudio realizado sobre tesis doctorales de enfermería, en las cuales el objeto de estudio de mayor interés corresponde a conocimientos del personal de enfermería sobre diversos aspectos relacionados con la práctica y la calidad de atención que brinda, condición que se ve afectada por las dificultades que se encuentran en las instituciones para la realización de investigaciones clínicas, principalmente por el tiempo que implica la realización de trámites a nivel institucional. Si bien estos últimos son necesarios para la observancia de principios éticos, debido a su complejidad, representan retrasos en la investigación, lo que repercute en el tiempo de obtención de resultados y por ende, en la conclusión del trabajo de tesis, situación que se refleja en la eficiencia terminal de los posgrados. Estas barreras son identificadas también por otros autores como Valencia ${ }^{18}$, reporta que los estudiantes de posgrado enfrentan dificultades para la elaboración de su trabajo de tesis, pueden ser intrínsecas, inherentes al programa educativo y extrínsecas que incluyen aspectos ligados a situaciones personales, familiares o económicas, así como habilidades o disposición de tiempo de los estudiantes.

Es necesario considerar las dificultades que representan las exigencias de organismos reguladores de estudios de posgrado como CONACYT o los Comités Interinstitucionales para la Evaluación de la Educación Superior (CIEES) que establecen tiempos acotados para que la conclusión del programa sea válida, lo que hace que los trabajos de investigación se tengan que realizar en población disponible y sobre temas que permitan la pronta obtención de resultados.

Lo anterior sugiere que si la investigación para la generación de evidencia científica en el área clínica con estudios experimentales es escasa, podría explicarse por factores ajenos al programa académico como son: largos periodos de tiempo que implica la obtención de permisos para realizar investigaciones en el ámbito hospitalario, la carencia de apoyos económicos o ayuda financiera para los estudiantes, escasa o nula colaboración multidisciplinar para el desarrollo de investigaciones de alto nivel o la limitada habilidad del estudiante.

En el ámbito científico el nivel de impacto de las tesis de grado y posgrado es bajo, la publicación de resultados es mínima así que el conocimiento generado pierde valor; sin embargo, el interés en el análisis de los productos de posgrado brinda una visión, aunque limitada, interesante para percibir la necesidad de la formación en investigación de los estudiantes y docentes del posgrado en enfermería. 
Las limitaciones del estudio radicaron principalmente en el reducido número de características incluidas en este, en la diferencia de años de duración de los programas y la diferencia del número de generaciones y estudiantes que han egresado de cada programa.

\section{CONCLUSIONES}

Es necesario prestar mayor atención a la selección de los temas de investigación de estudiantes de posgrado, motivarlos a identificar temáticas de investigación acordes al nivel de posgrado en el que se encuentren, las cuales se traduzcan en aportaciones valiosas para el ejercicio de la disciplina en el ámbito hospitalario, comunitario y educativo. Se requiere reforzar los aspectos de constancia y disciplina en el trabajo, lectura crítica en inglés y portugués, búsqueda bibliográfica en bases de datos de corte nacional e internacional para incrementar el acervo cultural científico de los estudiantes, además de ampliar su visión sobre problemas concernientes a la disciplina y las posibilidades de aportación de conocimiento.

A su vez, es indispensable que desde las coordinaciones de posgrado se organicen eventos intra e interdisciplinarios con la participación de diversas instituciones de educación superior, en los cuales se muestren avances de las investigaciones realizadas y sirvan como un espacio de convivencia y comunicación entre estudiantes de posgrado. Asimismo, es urgente diseñar estrategias para divulgar información sobre líneas de investigación existentes y definidas por la OMS.

La mejoría en la producción de conocimiento por estudiantes de posgrado en enfermería se vería beneficiada al apoyar a los alumnos en la publicación de sus resultados en revistas científicas, promoviendo la práctica de investigación significativa y desarrollando diseños de investigación secundaria como revisiones sistemáticas, revisiones críticas o meta-análisis, útiles para aplicarse como las Guías de Práctica Clínica.

Debido a la importancia que tiene la labor del facilitador, se debe pugnar por mantener una relación estrecha y productiva entre el tutor y el estudiante mediante la búsqueda bibliográfica intensa, recurriendo a revistas internacionales de alto impacto y promoviendo la utilización de referencias en idiomas distintos al español.

Dado que la enfermería tiene un gran componente social, sería necesario incrementar el interés en la realización de estudios de tipo cualitativo, explorando aspectos subjetivos en pacientes y en el personal de enfermería con el fin de mejorar la atención y el cuidado considerando aspectos filosóficos, epistemológicos e ideológicos desde este abordaje.

Hasta ahora el aporte al conocimiento que hace el personal de enfermería por los egresados de posgrado ha sido limitado debido a las barreras que existen en la investigación, a dificultades individuales y del entorno que se presentan para realizarla, mismas que marcan el éxito o fracaso. Por lo tanto, es imprescindible la gestión de apoyo económico para impulsar la multidisciplinariedad, mediante la colaboración entre estudiantes de la misma disciplina y de otras afines como medicina, nutrición, farmacología, fisioterapia o pedagogía de la misma universidad o de otras universidades nacionales e internacionales para incrementar la cantidad y calidad de la generación del conocimiento.

Si bien es cierto que no será posible eliminar o modificar los obstáculos en un corto plazo, es necesario considerar alternativas de solución como la inclusión de estudiantes en redes de investigación de la misma universidad o de otros estados o países, así como en las redes existentes de las diversas áreas del campo de la enfermería, además de promover la participación de estudiantes en investigaciones que cuenten con financiamiento y que sean desarrolladas por profesores de 
enfermería o de áreas afines, especialmente en el área de salud pública o la integración con equipos multidisciplinarios. Del mismo modo, el establecimiento de convenios de colaboración entre instituciones hospitalarias y de atención comunitaria, al igual que centros educativos y lugares de asistencia con la FE, favorecería el desarrollo de investigaciones y agilizaría los trámites administrativos para la pronta obtención de resultados y la consecuente presentación del trabajo de tesis.

\section{RESPONSABILIDADES ÉTICAS}

Protección de personas y animales. La recopilación de datos no involucró directamente a seres humanos.

Confidencialidad. Al conjuntar la información se omitió el nombre del autor y director de la tesis para respetar su anonimato.

Conflicto de intereses. Los autores declaran no tener conflicto de intereses.

Financiamiento. Ninguno.

\section{REFERENCIAS}

1. Borsinger-de Montemayor A, Cubo-de Severino L. Los textos de la ciencia: principales clases del discurso académico-científico. Córdoba, Argentina: Comunic-arte Editorial; 2005.

2. Castro-Rodríguez Y.Indicadores bibliométricos de las tesis sustentadas por estudiantes de Odontología, Perú. Edumecentro. 2018; 10(4): 1-19. https://bit.ly/2JrJ5qE

3. Ramírez-García RG, Pérez-Colunga BY, Soto-Bernabé AK, Mendoza-Tovar M, Coiffier-López FY, GleasonGuevara KJ, et al. Desarmando el rompecabezas en torno a la experiencia de elaboración de una tesis de maestría. Perf. educ. 2017; 39(155): 68-86. https://bit.ly/36j7RSx

4. Franco-CoffréJA, Piedra-Chavez KA, Cunalema-FernandezJA. Producción investigativa del pensamiento reflexivo y crítico del profesional de enfermería en el Ecuador. Rev. Publicando. 2018; $5(15$ (1)): 45164. https://bit.ly/2JrWrTP

5. Saltos-Llerena IJ, Rojas-Jaramillo RR, Guerrero-Onofa SJ, Herrera-Becerra KV. Importancia del pensamiento crítico en la Carrera de Enfermería de la Universidad Central del Ecuador, en el período abril-agosto 2017. Quito: UCE.; 2017. https://bit.ly/39gycso

6. Colegio de Postgraduados. Lineamientos para la operación de las líneas de generación y/o aplicación del conocimiento del Colegio de Postgraduados (LGAC-CP). Dictaminados en la $3^{\text {a }}$ Sesión Extraordinaria del COMERI. México: COLPOS-NIS-o019; 2016. https://bit.ly/3092mMn

7. Duarte-Hueros A, Guzmán-Franco MD, Yot-Domínguez CR. Aportaciones de la formación blended learning al desarrollo profesional docente. RIED. 2018; 21(1): 155-74.

8. Acuña SR, López-Aymes G. Análisis de la interacción estudiante-profesor en asesorías para el desarrollo de la tesis de posgrado. En: Roig-Vila, R (Ed.). El compromiso académico y social a través de la investigación e innovación educativas en la Enseñanza Superior. Barcelona: Octaedro; 2018.

9. Sánchez-Rodríguez JR, Aguayo-Cuevas CP, Galdames-Cabrera LG. Desarrollo del conocimiento de enfermería, en busca del cuidado profesional. Relación con la teoría critica. Rev. cuba. enferm. 2017; 33(3). https://bit.ly/3mp202b

10. Borges-Damas L, Sánchez-Machado R, Sixto-Pérez A, Valcárcel-Izquierdo N, Peñalver-Sinclaiy AG, Parra-Linares E. Teoría fundamentada en Datos como alternativa investigativa para significar el cuidado en la enfermería cubana. Rev. cuba. enferm. 2019; 35(3): 1-19. https://bit.ly/3lnAFhc 
11. Bispo-Hirano GS, Takáo-Lopes CT, Bottura-Leite de Barros AL. Development of research on nursing diagnoses in Brazilian graduate programs. Rev. Bras. Enferm. 2019; 72(4): 926-32.

https://doi.org/10.1590/0034-7167-2018-0259

12. Zárate-Grajales R. La investigación un desafío para la enfermería en la Región de las Américas. Enferm. univ. 2012; 9(4): 4-8. https://bit.ly/36ktMZO

13. Nájera RM, Castrillón-Agudelo MC. La Enfermería en América Latina. Situación actual, áreas críticas y lineamientos para un Plan de Desarrollo. México: Asociación Latinoamericana de Escuelas y Facultades de enfermería (ALADEFE).

14. Echeverría-Echeverría EI. Estrategia didáctica para estimular el aprendizaje con mentalidad reflexiva en la formación del estudiante que ingresa a la carrera de enfermería de la Universidad Regional Autónoma de Los Andes. Ecuador: UNIANDES; 2017.

15. De Souza CJ, Silvino ZR. The production of the professional master's degree in nursing of the Federal University of Santa Catarina, 2013-2016. Rev. Bras. Enferm. 2018; 71(Supl. 6): 2751-7.

http://dx.doi.org/10.1590/0034-7167-2018-0153

16. Silvan-Scochi CG, Lima-Gelbcke F, Ferreira MA, Dias da Silva-Lima MA, Grillo-Padilha K, ArtalPadovani N, et al. Nursing Doctorates in Brazil: research formation and theses production. Rev. Latino-Am. Enfermagem. 2015; 23(3): 387-94. http://dx.doi.org/10.1590/0104-1169.0590.2564

17. Partezani-Rodrigues RA, do Carmo Cruz-Robazzi ML, Lorenzini-Erdmann A, Dumet-Fernandes J, Bottura Leite-de Barros AL, Souza-Ramos FG. Doctoral theses from nursing postgraduate programs in Brazil and their association with the millennium development goals. Rev. Latino-Am. Enfermagem. 2015; 23(3): 395-403. http://dx.doi.org/10.1590/0104-1169.0667.2565

18. Valencia-Aguirre AC. Elaboración de tesis de posgrado en educación desde experiencias en procesos formativos. Diseminaciones. 2019; 2(3): 143-6o. https://bit.ly/2HOZZhE

19. Koepsell DR, Ruiz-de Chávez MH. Ética de la investigación. Integridad científica. México: Comisión Nacional de Bioética/Secretaría de Salud; 2015.

20. Chois-Lenis PM, Jaramillo-Echeverri LG. La investigación sobre la escritura en posgrado: estado del arte. Leng. 2016; 44(2): 227-59. https://bit.ly/2KV5T2L

21. Universidad Autónoma del Estado de Morelos. Facultad de Enfermería. Maestría en enfermería. Programa académico. México: UAEM; 2018.

22. Universidad Autónoma del Estado de Morelos. Reglamento General de Estudios de Posgrado. México: UAEM; 2018.

23. Rubio-Acuña M, Arias-Burgos M. Fenomenología y conocimiento disciplinar de enfermería. Rev. cuba. enferm. 2013; 29(3): 191-8. https://bit.ly/2JyBdnm

24. Marocco-Duran EC, Pellegrino-Toledo V. Análise da produção do conhecimento em processo de enfermagem: estudo exploratório-descritivo. Rev. Gaúcha Enferm. 2011; 32(2): 234-40.

https://doi.org/10.1590/S1983-14472011000200004 